\title{
Ligand-catalyzed Asymmetric Alkynylboration of Enones: A New Paradigm for Asymmetric Synthesis using Organoboranes
}

\author{
T. Robert Wu and J. Michael Chong* \\ Guelph-Waterloo Centre for Graduate Work in Chemistry and Biochemistry, \\ Department of Chemistry, University of Waterloo, Waterloo, Ontario, Canada \\ N2L $3 G 1$
}

\section{Supporting Information}

\section{General Experimental}

All reactions were performed using flame-dried glassware under an argon atmosphere. Dichloromethane was freshly distilled from $\mathrm{CaH}_{2}$. Diethyl ether was freshly distilled from $\mathrm{Na}$ /benzophenone. Diisopropyl alkynylboronates were prepared as reported by Brown. ${ }^{1}$ Chiral 3,3'-disubstituted binaphthols were synthesized using procedures described previously. ${ }^{2}{ }^{1} \mathrm{H}$ NMR and ${ }^{13} \mathrm{C}$ NMR spectra were recorded in $\mathrm{CDCl}_{3}$ at $300 \mathrm{MHz}$ and $75 \mathrm{MHz}$, respectively.

\section{General procedure for stoichiometric alkynylation of enones:}

Alkynylations of enones using stoichiometric amount of binaphthol-modified alkynylboronates were carried out according to a previous procedure: ${ }^{3}$ (S)-3,3' -Diiodo-2,2'-dihydroxy-1,1-binaphthyl (127 $\mathrm{mg}, \quad 0.236 \mathrm{mmol})$ and lithium triisopropyl $B$-1-alkynylborate $(0.232 \mathrm{mmol})$ were placed in a $25 \mathrm{~mL}$ round-bottomed flask under argon. THF $(5 \mathrm{~mL})$ was added slowly to the mixture at $0{ }^{\circ} \mathrm{C}$. The solution was stirred at $0{ }^{\circ} \mathrm{C}$ for $1 \mathrm{~h}$ then warmed up to room temperature for $5 \mathrm{~h}$. Solvent was evaporated under high vacuum. The enone (0.154 mmol) and $\mathrm{CH}_{2} \mathrm{Cl}_{2}(15 \mathrm{~mL})$ were added followed by $\mathrm{BF}_{3} \cdot \mathrm{OEt}_{2}(58 \mu \mathrm{L}, 0.461 \mathrm{mmol})$. The 
reaction was stirred at room temperature for $3 \mathrm{~h}$. After the reaction was complete, saturated $\mathrm{NH}_{4} \mathrm{Cl}$ (aq.) was added to quench the reaction. The organic layer was separated and the aqueous layer was washed with $\mathrm{CH}_{2} \mathrm{Cl}_{2}(2 \times 5 \mathrm{~mL})$. The combined organic phase was washed with water and dried over $\mathrm{Na}_{2} \mathrm{SO}_{4}$. Most of the binaphthol was collected by crystallization from $\mathrm{CH}_{2} \mathrm{Cl}_{2} /$ hexanes and the mother liquid was purified by column chromatography (acetone/hexane 1:10) to give the 1,4-addition product. Enantiomeric excesses of all products were determined by HPLC with a Chiralcel OD column. Absolute configurations for phenylacetylene and 1-octyne adducts to 1,3-bis(4-bromophenyl)-2-propen-1-one were determined by X-ray crystallography. ${ }^{3,4}$ The absolute configurations of other adducts have been assigned based on these known absolute configurations and the assumption that the enones are sufficiently similar in structure (all with $\beta$-aryl substituents) that the sense of asymmetric induction should be invariant.

\section{General procedure for catalytic alkynylation of enones (procedure A):}

A mixture of diisopropyl $B$-1-octynylboronate $(215 \mathrm{mg}, 0.9 \mathrm{mmol})$, enone $(0.3 \mathrm{mmol})$ and (S)-3,3' -diiodo-2,2'-dihydroxy-1,1-binaphthyl (32 mg, $0.06 \mathrm{mmol}$ ) were stirred in $\mathrm{CH}_{2} \mathrm{Cl}_{2}(4 \mathrm{~mL})$ under argon at room temperature for $6 \mathrm{~h}$, then brought to reflux for $24 \mathrm{~h}$. The reaction was quenched with saturated aqueous $\mathrm{NH}_{4} \mathrm{Cl}$. The organic phase was washed with brine and dried over $\mathrm{Na}_{2} \mathrm{SO}_{4}$. Purification was done by column chromatography (acetone/hexane 1:10) on silica gel. The chiral ligand was recovered in quantitative yield.

\section{One-pot procedure for catalytic alkynylation of enones from 1-alkyne (procedure B):}

To a solution of 1-alkyne $(1.0 \mathrm{mmol})$ in $\mathrm{Et}_{2} \mathrm{O}(5 \mathrm{~mL})$ was added $0.63 \mathrm{~mL}$ of $n$-BuLi (1.0 mmol, 1.6 M) dropwise at $-78{ }^{\circ} \mathrm{C}$. The resulting solution was stirred at that temperature for 20 minutes then added dropwise to a mixture of $\left({ }^{i} \mathrm{PrO}\right)_{3} \mathrm{~B}$ in $5 \mathrm{mLEt}_{2} \mathrm{O}$ at $-78^{\circ} \mathrm{C}$. The suspension was stirred at 
$-78{ }^{\circ} \mathrm{C}$ for $2 \mathrm{~h}$ and warmed up to room temperature for $1 \mathrm{~h}$. The reaction was again cooled to $-78^{\circ} \mathrm{C}$, and $0.5 \mathrm{~mL}$ anhydrous $\mathrm{HCl}$ in $\mathrm{Et}_{2} \mathrm{O}(2 \mathrm{M}, 1 \mathrm{mmol})$ was added slowly. After the reaction was allowed to warm to room temperature, volatiles were pumped off under high vacuum. To the yellowish oily residue was added $\mathrm{CH}_{2} \mathrm{Cl}_{2}(4 \mathrm{~mL})$, enone $(0.3 \mathrm{mmol})$ and catalyst $(0.06 \mathrm{mmol})$ at room temperature. The mixture was stirred at ambient temperature for $6 \mathrm{~h}$ then brought to reflux for $24 \mathrm{~h}$. Workup was the same as procedure A.

\section{(S)-1,3-Diphenyl-4-undecyn-1-one (7a)}

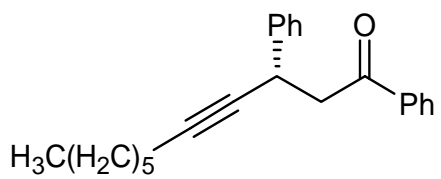

$[\alpha]_{\mathrm{D}}=+31.4\left(\mathrm{c} 2.04, \mathrm{CHCl}_{3}, 85 \%\right.$ ee $)$; IR (neat): $\mathrm{C}=\mathrm{O} 1684 \mathrm{~cm}^{-1} ;{ }^{1} \mathrm{H}$ NMR $\left(300 \mathrm{MHz}, \mathrm{CDCl}_{3}\right)$ $\delta 0.86(3 \mathrm{H}, \mathrm{t}, \mathrm{J}=6.7 \mathrm{~Hz}), 1.15-1.48(8 \mathrm{H}, \mathrm{m}), 2.14(2 \mathrm{H}, \mathrm{dt}, \mathrm{J}=2.2 \mathrm{~Hz}, 6.8 \mathrm{~Hz}), 3.26(1 \mathrm{H}, \mathrm{dd}, \mathrm{J}=$ 16.4Hz, 6Hz), $3.52(1 \mathrm{H}, \mathrm{dd}, \mathrm{J}=16.4 \mathrm{~Hz}, 8.3 \mathrm{~Hz}), 4.35-4.40(1 \mathrm{H}, \mathrm{m}), 7.18-7.94(10 \mathrm{H}, \mathrm{m}) ;{ }^{13} \mathrm{C} \mathrm{NMR}$ $\left(\mathrm{CDCl}_{3}\right) \delta 197.3,141.9,136.9,132.9,128.4,128.0,127.4,126.6,83.5,80.9,47.6,33.3,31.2,28.7$, 28.4, 22.4, 18.7, 13.9; MS (EI), m/e (relative intensity) 318 (1, $\left.\mathrm{M}^{+}\right), 247$ (13), 233 (33), 128 (20), 105 (100), 91 (27), 77 (53). Anal. Calcd for $\mathrm{C}_{23} \mathrm{H}_{26} \mathrm{O}$ : C, 86.75; H, 8.23. Found C, 86.55; H, 8.20. \%ee was determined by HPLC (hexane/iPrOH $=99.9 / 0.1$, flow rate $=0.5 \mathrm{~mL} / \mathrm{min}), \mathrm{t}_{\mathrm{R}}=55 \mathrm{~min}(S)$, $\mathrm{t}_{\mathrm{R}}=61 \min (R)$.

\section{(R)-3-Naphthalen-1-yl -1-phenyl-4-undecyn-1-one (7b)}<smiles>CCCC#C[C@H](CC(=O)c1ccccc1)c1cccc2ccccc12</smiles> 
$[\alpha]_{\mathrm{D}}=-13.1$ (c 1.39, $\mathrm{CHCl}_{3}, 96 \%$ ee); IR (neat): $\mathrm{C}=\mathrm{O} 1684 \mathrm{~cm}^{-1} ;{ }^{1} \mathrm{H} \mathrm{NMR}\left(300 \mathrm{MHz}, \mathrm{CDCl}_{3}\right)$ $\delta 0.87(3 \mathrm{H}, \mathrm{t}, \mathrm{J}=6.9 \mathrm{~Hz}), 1.12-1.47(8 \mathrm{H}, \mathrm{m}), 2.17(2 \mathrm{H}, \mathrm{dt}, \mathrm{J}=2.2 \mathrm{~Hz}, 6.8 \mathrm{~Hz}), 3.32(1 \mathrm{H}, \mathrm{dd}, \mathrm{J}=$

$16.8 \mathrm{~Hz}, 3.9 \mathrm{~Hz}), 3.65(1 \mathrm{H}, \mathrm{dd}, \mathrm{J}=16.8 \mathrm{~Hz}, 9.6 \mathrm{~Hz}), 5.18-5.22(1 \mathrm{H}, \mathrm{m}), 7.43-8.18(12 \mathrm{H}, \mathrm{m}) ;{ }^{13} \mathrm{C}$ NMR $\left(\mathrm{CDCl}_{3}\right) \delta 197.6,137.4,137.0,134.0,133.0,129.0,128.5,128.2,127.6,126.2,125.5,125.4$, 125.2, 123.0, 84.1, 80.8, 46.5, 31.2, 30.0, 28.7, 28.4, 22.4, 18.8, 14.0; MS (EI), m/e (relative intensity) $368\left(3, \mathrm{M}^{+}\right), 284$ (23), 283 (83), 179 (48), 178 (32), 165 (30), 105 (100). Anal. Calcd for $\mathrm{C}_{27} \mathrm{H}_{28} \mathrm{O}: \mathrm{C}, 88.00 ; \mathrm{H}$, 7.66. Found $\mathrm{C}, 88.10 ; \mathrm{H}$, 7.58. \%ee was determined by HPLC (hexane/PrOH $=99.5 / 0.5$, flow rate $=1 \mathrm{~mL} / \mathrm{min}), \mathrm{t}_{\mathrm{R}}=17 \mathrm{~min}(S), \mathrm{t}_{\mathrm{R}}=24 \mathrm{~min}(R)$. Note that the difference in absolute configuration between $\mathbf{7 a}$ and $\mathbf{7 b}$ is due to differences in CIP priorities: 1-naphthyl >1-octynyl > phenyl.

\section{(R)-3-(2-Furanyl)-1-phenyl-4-undecyn-1-one (7c)}

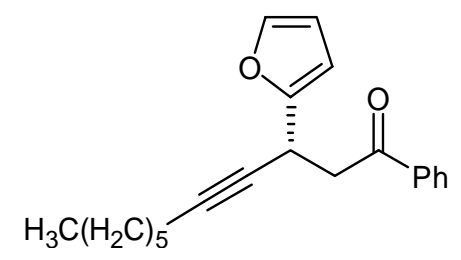

$[\alpha]_{\mathrm{D}}=-10.4$ (c 2.0, $\mathrm{CHCl}_{3}, 88 \%$ ee); IR (neat): $\mathrm{C}=\mathrm{O} 1689 \mathrm{~cm}^{-1} ;{ }^{1} \mathrm{H} \mathrm{NMR}\left(300 \mathrm{MHz}, \mathrm{CDCl}_{3}\right) \delta 0.84$ $(3 \mathrm{H}, \mathrm{t}, \mathrm{J}=6.9 \mathrm{~Hz}), 1.10-1.50(8 \mathrm{H}, \mathrm{m}), 2.09-2.15(2 \mathrm{H}, \mathrm{dt}, \mathrm{J}=2.4 \mathrm{~Hz}, 6.9 \mathrm{~Hz}), 3.43-3.46(2 \mathrm{H}, \mathrm{m})$, 4.43-4.49 (1H, m), $6.22(1 \mathrm{H}, \mathrm{dd}, \mathrm{J}=3.3 \mathrm{~Hz}, 0.9 \mathrm{~Hz}), 6.27(1 \mathrm{H}, \mathrm{dd}, \mathrm{J}=3.3 \mathrm{~Hz}, 1.9 \mathrm{~Hz}), 7.30(1 \mathrm{H}, \mathrm{dd}$, $\mathrm{J}=0.9 \mathrm{~Hz}, 1.9 \mathrm{~Hz}), 7.39-7.60(5 \mathrm{H}, \mathrm{m}), 7.90-8.02(2 \mathrm{H}, \mathrm{m}) ;{ }^{13} \mathrm{C} \mathrm{NMR}\left(\mathrm{CDCl}_{3}\right) \delta 196.9,154.1,136.9$, $133.1,128.5,128.2,110.3,105.9,82.9,78.3,43.8,31.3,28.7,28.4,27.2,22.5,18.7,14.0 ; \mathrm{MS}(\mathrm{EI})$, m/e (relative intensity) $308\left(1, \mathrm{M}^{+}\right), 105$ (100), 77 (43). Anal. Calcd for $\mathrm{C}_{21} \mathrm{H}_{24} \mathrm{O}_{2}: \mathrm{C}, 81.78 ; \mathrm{H}, 7.84$. Found C, 81.90; H, 7.76. \%ee was determined by HPLC (hexane/iPrOH $=99.8 / 0.2$, flow rate $=0.5$ $\mathrm{mL} / \min ), \mathrm{t}_{\mathrm{R}}=34 \min (R), \mathrm{t}_{\mathrm{R}}=36 \min (S)$. 


\section{(S)-4-Phenyl-5-dodecyn-2-one (7d)}

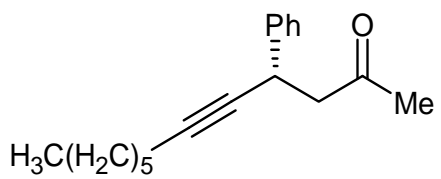

$[\alpha]_{\mathrm{D}}=+2.4\left(\mathrm{c} 1.4, \mathrm{CHCl}_{3}, 94 \%\right.$ ee $) ; \mathrm{IR}$ (neat): $\mathrm{C}=\mathrm{O} 1706 \mathrm{~cm}^{-1} ;{ }^{1} \mathrm{H} \mathrm{NMR}\left(300 \mathrm{MHz}, \mathrm{CDCl}_{3}\right) \delta 0.87$ $(3 \mathrm{H}, \mathrm{t}, \mathrm{J}=6.9 \mathrm{~Hz}), 1.26-1.49(8 \mathrm{H}, \mathrm{m}), 2.11(3 \mathrm{H}, \mathrm{s}), 2.15-2.19(2 \mathrm{H}, \mathrm{dt}, \mathrm{J}=2.1 \mathrm{~Hz}, 6.9 \mathrm{~Hz}), 2.69(1 \mathrm{H}$, $\mathrm{dd}, 16.1 \mathrm{~Hz}, 6.1 \mathrm{~Hz}), 2.86(1 \mathrm{H}, \mathrm{dd}, \mathrm{J}=16.1 \mathrm{~Hz}, 8.5 \mathrm{~Hz}), 4.10-4.15(1 \mathrm{H}, \mathrm{m}), \delta 7.17-7.40(5 \mathrm{H}, \mathrm{m}) ;{ }^{13} \mathrm{C}$ $\operatorname{NMR}\left(\mathrm{CDCl}_{3}\right) \delta 206.1,141.6,128.5,127.3,126.8,83.6,80.6,52.3,33.2,31.3,30.6,28.9,28.5$, 22.5, 18.8, 14.0; MS (EI), m/e (relative intensity) $256\left(1, \mathrm{M}^{+}\right), 185$ (52), 171 (49), 129 (39), 128 (41), 43 (100). Anal. Calcd for $\mathrm{C}_{18} \mathrm{H}_{24} \mathrm{O}$ : C, 84.32; H, 9.44. Found C, 84.26; H, 9.32. \%ee was determined by HPLC (hexane/iPrOH $=99.9 / 0.1$, flow rate $=0.5 \mathrm{~mL} / \mathrm{min}$ ), $\mathrm{t}_{\mathrm{R}}=54 \mathrm{~min}(R), \mathrm{t}_{\mathrm{R}}=60$ $\min (S)$.

(S)-1,3,5-Triphenyl-4-pentyn-1-one (7e)

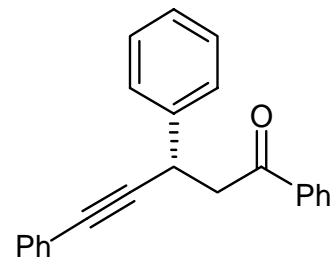

$[\alpha]_{\mathrm{D}}=+30.1\left(\mathrm{c} 1.44, \mathrm{CHCl}_{3}, 82 \% \mathrm{ee}\right) ; \mathrm{IR}(\mathrm{KBr}): \mathrm{C}=\mathrm{O} 1680 \mathrm{~cm}^{-1} ;{ }^{1} \mathrm{H}$ NMR $\left(300 \mathrm{MHz}, \mathrm{CDCl}_{3}\right)$ $\delta 3.39(1 \mathrm{H}, \mathrm{dd}, \mathrm{J}=16.5 \mathrm{~Hz}, 6.2 \mathrm{~Hz}), 3.65(1 \mathrm{H}, \mathrm{dd}, \mathrm{J}=16.5 \mathrm{~Hz}, 7.8 \mathrm{~Hz}), 4.63(1 \mathrm{H}, \mathrm{dd}, \mathrm{J}=6.2 \mathrm{~Hz}$, 7.8Hz), 7.31-8.05 (15H, m); ${ }^{13} \mathrm{C}$ NMR $\left(\mathrm{CDCl}_{3}\right) \delta 197.0,141.2,136.8,133.1,131.6,128.6,128.5$, 128.1, 127.8, 127.5, 127.0, 123.3, 90.7, 83.3, 47.2, 33.7; MS (EI), m/e (relative intensity) 310 (10, $\mathrm{M}^{+}$), 205 (100), 191 (44), 105 (71), 77 (57). Anal. Calcd for $\mathrm{C}_{23} \mathrm{H}_{18} \mathrm{O}$ : C, 89.00; H, 5.84. Found C, 89.13; $\mathrm{H}, 5.83$. \%ee was determined by HPLC (hexane $/ \mathrm{PrOH}=99.8 / 0.2$, flow rate $=0.5 \mathrm{~mL} / \mathrm{min}$ ), $\mathrm{t}_{\mathrm{R}}=100 \min (R), \mathrm{t}_{\mathrm{R}}=115 \min (S)$. 


\section{(R)-3-(1-Naphthalenyl)-1,5-diphenyl-4-pentyn-1-one (7f)}

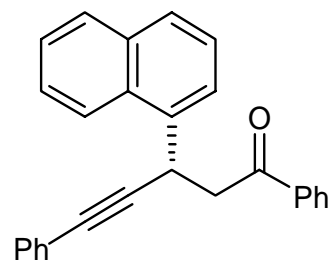

$[\alpha]_{\mathrm{D}}=+37.3\left(\mathrm{c} 1.01, \mathrm{CHCl}_{3}, 90 \% \mathrm{ee}\right) ; \mathrm{IR}(\mathrm{KBr}): \mathrm{C}=\mathrm{O} 1686 \mathrm{~cm}^{-1} ;{ }^{1} \mathrm{H} \mathrm{NMR}\left(300 \mathrm{MHz}, \mathrm{CDCl}_{3}\right)$ $\delta 3.45(1 \mathrm{H}, \mathrm{dd}, \mathrm{J}=16.8 \mathrm{~Hz}, 4.2 \mathrm{~Hz}), 3.81(1 \mathrm{H}, \mathrm{dd}, \mathrm{J}=16.8 \mathrm{~Hz}, 9.6 \mathrm{~Hz}), 5.42(1 \mathrm{H}, \mathrm{dd}, \mathrm{J}=9.6 \mathrm{~Hz}$ 4.2Hz), 7.33-8.30 (17H, m); ${ }^{13} \mathrm{C}$ NMR $\left(\mathrm{CDCl}_{3}\right) \delta 197.2,136.8,136.7,134.0,133.2,130.3,129.0$, $128.6,128.2,128.1,127.9,127.8,126.4,125.7,125.5,125.4,123.4,122.9,90.6,83.8,46.2,30.4$; MS (EI), m/e (relative intensity) $360\left(11, \mathrm{M}^{+}\right), 255$ (35), 207 (100). Anal. Calcd for $\mathrm{C}_{27} \mathrm{H}_{20} \mathrm{O}: \mathrm{C}$, 89.97; H, 5.59. Found C, 90.05; H, 5.58. \%ee was determined by HPLC (hexane/iPrOH = 99/1, flow rate $=1 \mathrm{~mL} / \mathrm{min}), \mathrm{t}_{\mathrm{R}}=17 \min (S), \mathrm{t}_{\mathrm{R}}=22 \mathrm{~min}(R)$. Note that the difference in absolute configuration between 7e and 7f is due to differences in CIP priorities: 1-naphthyl > phenylethynyl $>$ phenyl.

\section{(S)-6-Benzyloxy-1,3-diphenyl-4-hexyn-1-one (7g)}

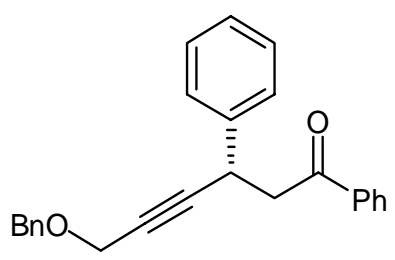

$[\alpha]_{\mathrm{D}}=-1.5$ (c 1.6, $\mathrm{CHCl}_{3}, 86 \%$ ee); IR (neat): 3062, 3030, 2232, 1684, 1598, 1495, 1450, 1352, 1255, 1205, 1072, $1035 \mathrm{~cm}^{-1} ;{ }^{1} \mathrm{H}$ NMR (300 MHz, $\left.\mathrm{CDCl}_{3}\right) \delta 3.33(1 \mathrm{H}, \mathrm{dd}, \mathrm{J}=16.8 \mathrm{~Hz}, 6 \mathrm{~Hz}), 3.58$ $(1 \mathrm{H}, \mathrm{dd}, \mathrm{J}=16.8 \mathrm{~Hz}, 7.8 \mathrm{~Hz}), 4.16-4.17(2 \mathrm{H}, \mathrm{m}), 4.46-4.53(3 \mathrm{H}, \mathrm{m}), 7.26-7.99(15 \mathrm{H}, \mathrm{m}) ;{ }^{13} \mathrm{C} \mathrm{NMR}$ $\left(\mathrm{CDCl}_{3}\right) \delta 196.8,140.9,137.5,136.7,133.2,128.6,128.5,128.3,128.1,128.0,127.7,127.5,127.0$ 87.9, 78.7, 71.2, 57.5, 47.0, 33.0; MS (EI), m/e (relative intensity) 354 (1, $\left.\mathrm{M}^{+}\right), 105$ (100), 77 (31). Anal. Calcd for $\mathrm{C}_{25} \mathrm{H}_{22} \mathrm{O}_{2}$ : C, 84.72; H, 6.26. Found C, 84.98; H, 6.17. \%ee was determined by 
HPLC (hexane $/ \mathrm{iPrOH}=97 / 3$, flow rate $=1 \mathrm{~mL} / \mathrm{min}), \mathrm{t}_{\mathrm{R}}=16 \min (R), \mathrm{t}_{\mathrm{R}}=26 \min (S)$.

(S)-6-Benzyloxy-3-naphthyl-1-phenyl-4-hexyn-1-one (7h)

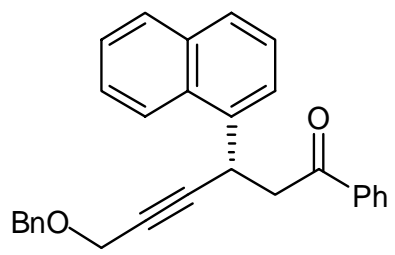

$[\alpha]_{\mathrm{D}}=+10.5$ (c $0.84, \mathrm{CHCl}_{3}, 95 \%$ ee); IR (neat): $\mathrm{C}=\mathrm{O} 1683 \mathrm{~cm}^{-1} ;{ }^{1} \mathrm{H}$ NMR $\left(300 \mathrm{MHz}, \mathrm{CDCl}_{3}\right)$

$\delta 3.41(1 \mathrm{H}, \mathrm{dd}, \mathrm{J}=17.1 \mathrm{~Hz}, 4.2 \mathrm{~Hz}), 3.75(1 \mathrm{H}, \mathrm{dd}, \mathrm{J}=17.1 \mathrm{~Hz}, 6.6 \mathrm{~Hz}), 4.18-4.19(2 \mathrm{H}, \mathrm{m}), 4.52(2 \mathrm{H}, \mathrm{s})$,

5.27-5.32 (1H, dd, J = 4.2Hz, 6.6Hz), 7.26-8.24 (17H, m); ${ }^{13} \mathrm{C} \mathrm{NMR}\left(\mathrm{CDCl}_{3}\right) \delta 197.1,137.5,136.7$, $136.4,134.1,133.3,130.3,129.1,128.6,128.3,128.2,128.1,128.0,127.7,126.4,125.7,125.5$, 125.3, 122.9, 87.9, 79.4, 71.2, 57.5, 46.0, 38.8, 29.8; MS (EI), m/e (relative intensity) $404\left(1, \mathrm{M}^{+}\right)$, 296 (17), 105 (100), 91 (33), 77 (33). Anal. Calcd for $\mathrm{C}_{29} \mathrm{H}_{24} \mathrm{O}_{2}$ : C, 86.11; H, 5.98. Found C, 85.99; $\mathrm{H}, 5.91$. \%ee was determined by HPLC (hexane $/ \mathrm{PrOH}=99 / 1$, flow rate $=1 \mathrm{~mL} / \mathrm{min}$ ), $\mathrm{t}_{\mathrm{R}}=58 \mathrm{~min}$ $(R), \mathrm{t}_{\mathrm{R}}=63 \min (S)$.

\section{References}

1. Brown, H. C.; Bhat, N. G..; Srebnik, M. Tetrahedron Lett. 1988, 29, 2631-2634.

2. Wu, T. R.; Shen, L. X.; Chong, J. M. Org. Lett. 2004, 6, 2701-2704.

3. Chong, J. M.; Shen, L. X.; Taylor, N. J. J. Am. Chem. Soc. 2000, 122, 1822-1823.

4. Shen, L. X. Ph.D. Dissertation, University of Waterloo, 2000 